\title{
Heart rate response to "off-road" running events in female athletes
}

\author{
U Creagh, T Reilly, A M Nevill
}

\begin{abstract}
Introduction-Despite the growing popularity of off-road running events, little information is available about the physiological stress of such activities. The demands of such events are unique in terms of the rough surface of the terrain encountered as well as the underfoot vegetation and the gradient.

Objectives-The aim of this study was to investigate the physiological response of female athletes, as measured by heart rate, to three common off-road running events: cross country running $(n=15)$, fell running $(n=20)$, and orienteering $(n=25)$. Methods-Heart rate responses were recorded during cross country and fell races, and orienteering by means of short range radiotelemetry. Road running ( $n=$ 21) was also studied as a reference.

Results-The mean heart rates for each event varied with the differing demands of the terrain. The highest (182 (10) beats/ minute; mean (SD)) was for road running and the lowest (172 (10) beats/minute) for orienteering. Orienteering evoked a significantly more variable response than all other events $\left(F_{4,100}=112.4 ; p<0.01\right)$, with a range of 44 beats/minute (142-186 beats/ minute) in the heart rate. This may be due to both the variability of the terrain and the additional technical aspect of this sport. There was a positive trend in the slope of the regression lines of heart rate against time for both road and cross country running, which was not evident in the fell runners or the orienteers. The latter events demonstrated no consistent pattern.
\end{abstract}

Conclusions-It is concluded that running off-road elicits a heart rate response that varies with the altering demands of surface, vegetation, and gradient.

(Br f Sports Med 1998;32:34-38)

Exercise Sciences,

Liverpool John Moores

University, Liverpool,

United Kingdom

U Creagh

T Reilly

A M Nevill

Correspondence to: Dr U Creagh, Centre for

Sport and Exercise Sciences, School of Human Sciences, Liverpool John Moores

University, Mountford

Building, Byrom Street,

Liverpool L3 3AF, United

Kingdom.

Accepted for publication 1 October 1997

Running off-road exposes the body to unfamiliar and often irregular stresses. This stress may be a result of the terrain surface encountered, the underfoot vegetation, and/or the gradient involved. The energy cost of running over rough/compliant terrain has been shown to be greater than that over good level surfaces. ${ }^{12}$ The level of energy expended on these terrain conditions is likely to fluctuate, even when the running velocity is constant. For example, running through deep vegetation places demands on many muscle groups not normally associated with road running (resulting from high knee lift, uneven stride patterns, a need to maintain balance, and so on), and also increases the demand on the regularly used musculature. Cross country running generally takes place on grass, with mud being a primary disturbance. In fell running, the terrain is considerably more compliant and the gradient has a strong influence on the energy expenditure. Orienteering terrain is likely to be the most varied, particularly in terms of underfoot vegetation, although the gradient also varies considerably, and the constant changing of direction in negotiating obstacles is also likely to interfere with running style consequently affecting recorded heart rates.

The linear relation between work load, heart rate, and oxygen consumption has frequently been used to estimate exercise intensity in field situations, where respiratory gas analysis may not be appropriate or feasible..$^{3-5}$ In the light of previous findings, ${ }^{6}$ the use of heart rate provides an acceptable estimate of energy expenditure for running over varied positive gradients (up to $4 \%$ ). However, the influence of steep gradients has not been established and there is some evidence that negative grade walking may alter the relationship between heart rate and oxygen consumption ${ }^{78}$ (implying a similar effect for running). Given such limitations, the aim of the current study was to investigate the pattern of the heart rate response under the different terrain conditions.

\section{Methods}

The heart rate responses to races over four different surfaces were compared. Road races were taken as the reference condition, while cross country, fell, and orienteering races were also examined. The data for each event were collected during a selection of different races. The nature of both road and cross country races is such that there is little difference between courses. The fell races and orienteering races, on the other hand, were observed over a wide range of courses in order to include a broad range of typical conditions.
SUBJECTS

The orienteering subjects were female elite level competitors (members of various national squads; $n=25$ ) of mean (SD) age 26.5 (5.0) years. All other subjects were at least club level in their own discipline. Local road runners $(\mathrm{n}=$ 21) were observed during $8-10 \mathrm{~km}$ races country runners $(n=15)$ were recruited at championship level races (mean (SD) age 26.9 (mean (SD) age 29.0 (6.2) years). The cross 
(8.0) years), while the fell runners $(n=20)$ were participants in local races of various length (mean (SD) age 30.1 (6.2) years).

\section{PROCEDURES}

Subjects wore a short range telemetry device (PE3000; Polar Sportstester, Kempele, Finland) for the measurement of heart rate during competitions, recording every $15 \mathrm{~s}$. Before the start of testing, all subjects were familiarised with the use of the heart rate monitor, including the fitting of the transmitter and the functions of the receiver. They were offered the option of wearing the transmitter on a chest strap or fitted to a specially designed sports bra (Polar Sportstester) for comfort.

\section{STATISTICAL ANALYSIS}

The results were analysed descriptively to establish mean, peak, and range of heart rates. The variability of the heart rates in the different sports was then compared. All heart rate profiles were analysed only from the time "steady state" was reached. This was determined visually from the heart rate profiles and was then verified with regression analysis. Steady state was reached within 5-15 minutes depending on the event; this determined steady state condition disregarded the relative variability around the mean value. The trends shown in the slopes of the established regression lines were then investigated. To establish the difference in the variability of heart rates between the various sports investigated, a one way analysis of variance was performed. This was carried out on the logarithm of the variances, as proposed by Scheffé as a test of homogeneity of variances which was relatively insensitive to departures from normality. ${ }^{9}$ To verify the use of logarithms, normality plots were established for both the residuals of the variances and the residuals of the logarithms. Post hoc tests (Tukey) were performed to isolate any significant differences.

\section{Results}

Table 1 provides a descriptive summary of the heart rates for each event. The mean heart rate of 172 beats/minute for the orienteering group corresponded to $88.7 \%$ of the mean age predicted maximum (220 - age) for that group. On average the highest heart rates were 186 beats/minute which was equivalent to $95.9 \%$ of the age predicted maximum of the group. The orienteers' mean relative effort level (in terms of percentage of age predicted maximum heart rate) was generally lower than in the other disciplines. Competitors in the other sports investigated were operating at greater than $90 \%$ of their predicted maximum heart rate throughout the duration of their events: road running $(95.3 \%)$, cross country $(93.2 \%)$, and fell running $(93 \%)$. When the mean heart rates were examined in relation to the maximum exercise heart rate recorded in each event, the orienteers were working at $92 \%$ of the maximum, whereas participants in the other events were exercising at a mean heart
Table 1 Descriptive data on heart rates in female endurance running events

\begin{tabular}{lrrrr}
\hline & \multicolumn{4}{l}{ Heart rate (beats/minute) } \\
\cline { 2 - 5 } & Road & Cross country & Fell & Orienteering \\
\hline Mean & 182 & 180 & 176 & 172 \\
Standard & & 8 & 8 & 10 \\
$\quad$ deviation & 10 & 8 & 163 & 142 \\
Minimum & 170 & 174 & 186 & 186 \\
Raximum & 190 & 187 & 23 & 44 \\
\hline
\end{tabular}

Table 2 Mean heart rate distribution during each running event (values expressed as \% of time in each heart rate band)

\begin{tabular}{lrrrl}
\hline \multirow{2}{*}{$\begin{array}{l}\text { Heart rate } \\
\text { (beats/minute) }\end{array}$} & \multicolumn{4}{l}{ Distribution (\%) } \\
\cline { 2 - 5 } & Road & Cross country & Fell & Orienteering \\
\hline$<160$ & 0.3 & 0.0 & 3.7 & 15.9 \\
$160-169$ & 11.6 & 4.0 & 29.5 & 27.9 \\
$170-179$ & 26.0 & 52.6 & 35.5 & 24.3 \\
$\geqslant 180$ & 62.1 & 43.4 & 31.2 & 31.9 \\
\hline
\end{tabular}

rate that was greater than $95 \%$ of the maximal exercise rate.

The mean distribution of the heart rates in orienteering and fell running showed a broader range of intensity in these events (see table 2). During both road running and cross country running, subjects' heart rates were generally higher, with little time spent at a heart rate below 170 beats/minute (10\% and $4 \%$ respectively). This is in contrast with the other events (44\% in orienteering and $33 \%$ in fell running) in which much of competition time is spent at lower heart rates.

The distribution of the heart rates during the events studied shows differences in the variances. The heart rates of all subjects varied considerably throughout the duration of orienteering and fell running events. Subjects participating in road and cross country races maintained more consistent heart rate profiles throughout their respective events. This was particularly apparent when visual comparison was made between the heart rate profiles of individuals during different events. Figure 1 shows a typical example from each sport.

Following the initial descriptive analysis, regression lines were fitted to each individual heart rate profile (fig 2). These regression lines were then compared to assess the direction of the slopes. The variances about the individual regression lines were then analysed and the degree of variability between the different running events compared. Examination of the slopes of the regression lines of all heart rate profiles showed that the orienteers maintained a mean heart rate that was relatively constant throughout their events, thereby maintaining a steady overall energy expenditure. There was a trend among road runners and cross country runners to increase the intensity of effort gradually towards the end of their races, resulting in a positive slope in the regression lines of their heart rate profiles. In orienteering there were as many subjects who produced a negative or neutral (no increase or decrease) trend as those whose heart rates increased throughout 

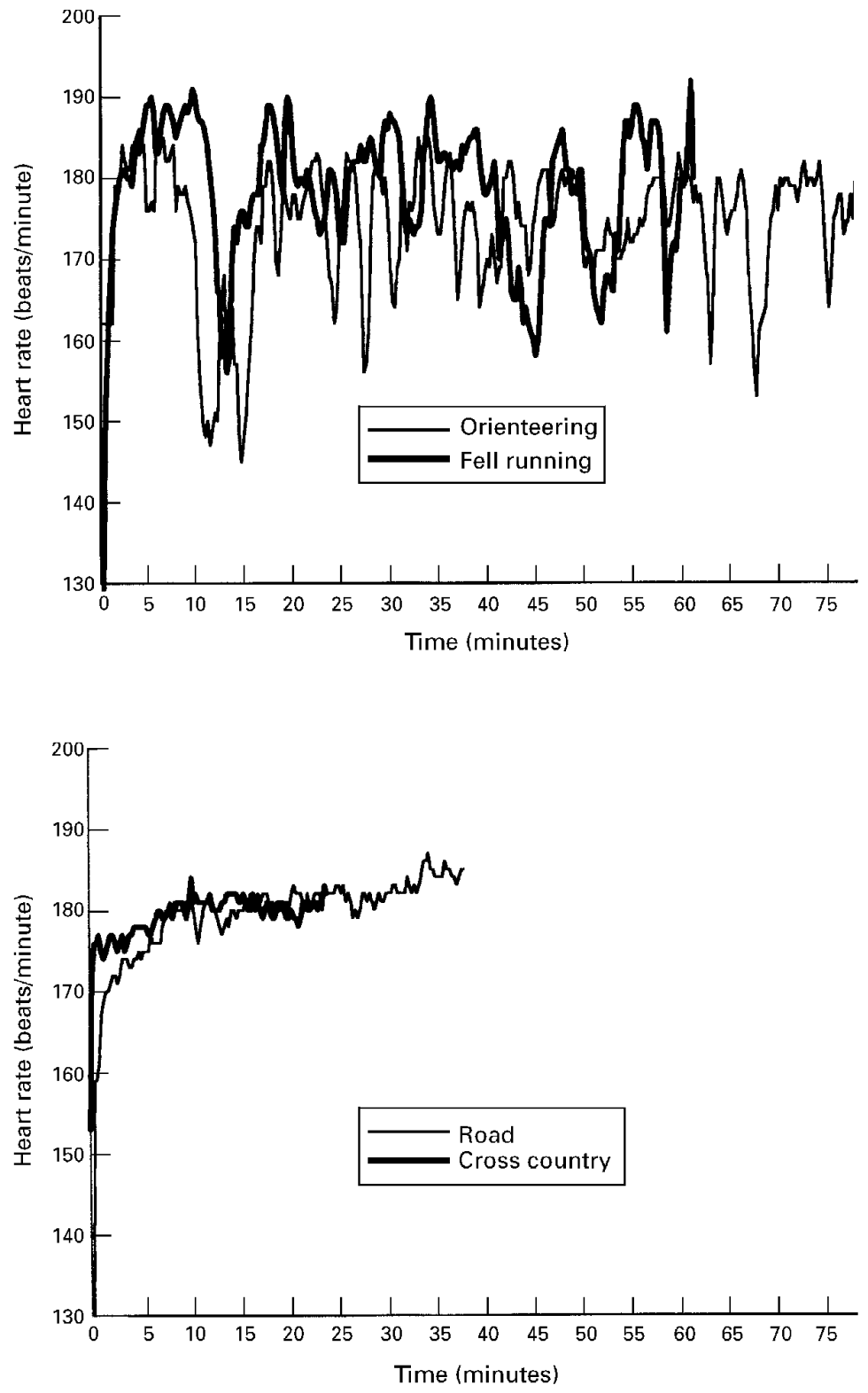

Figure 1 Typical heart rate profiles for each running event.

their event, while the fell runners showed an equal number of negative and positive trends (fig 3).

A one way analysis of variance was carried out on the logarithms of all variances (around the previously established regression lines) in order to make a statistical comparison of the variability in heart rates. An Anderson-Darling normality tes $\mathrm{t}^{10}$ verified that the residuals of the untreated variances were not normally distributed $\left(\mathrm{A}^{2}=5.425 ; \mathrm{p}<0.05\right)$, but after calculation of the logarithms a normal distribution of their residuals was evident $\left(\mathrm{A}^{2}=0.645 ; \mathrm{p}>0.05\right)$. This further supported the use of logarithms in differentiating between the running events. The analysis of variance showed that there was a significant difference in the variability of the heart rates between the events $\left(F_{4,100}=112.4\right.$; $\mathrm{p}<0.01)$. A Tukey HSD post hoc test indicated that there was a significant difference in the variance of the heart rates between orienteering
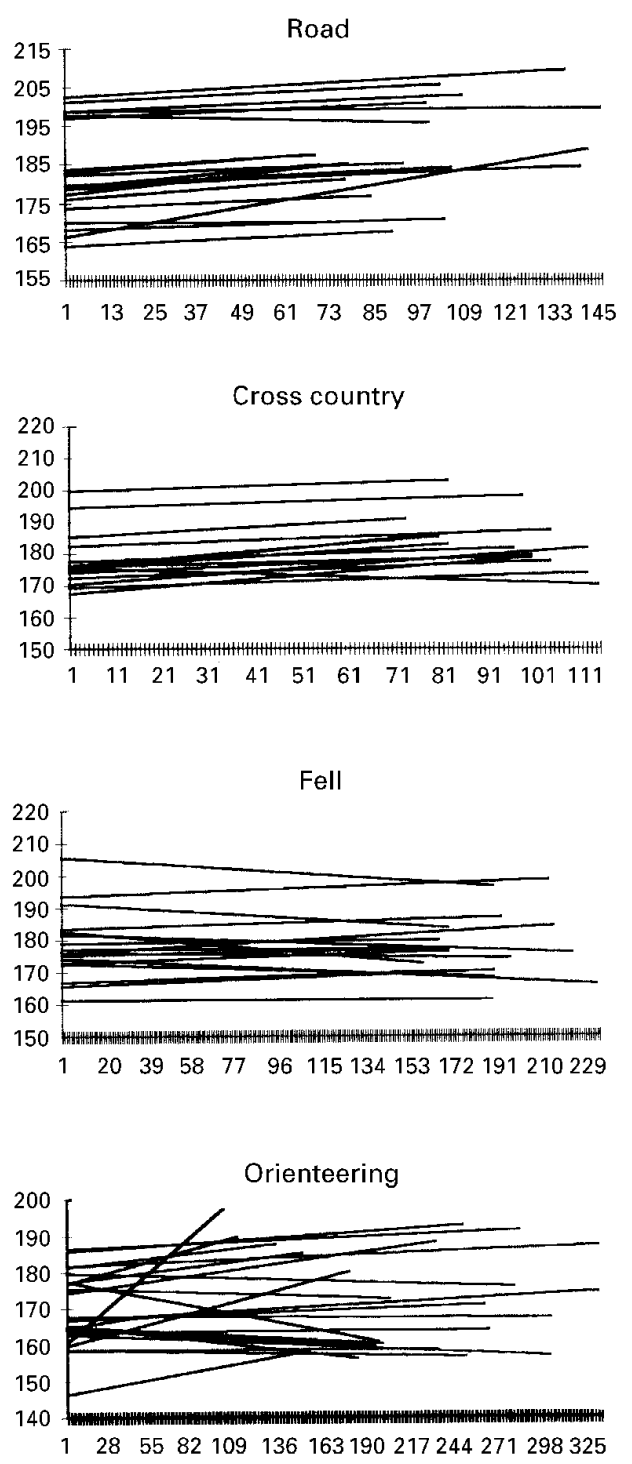

Figure 2 Heart rate regression lines for each running event.

and all other events studied. Although fell running displayed a large degree of variability, there was no significant difference in the variance of the heart rate profiles between fell racing and those during road and cross country racing. Table 3 indicates the mean variance in heart rates for each event.

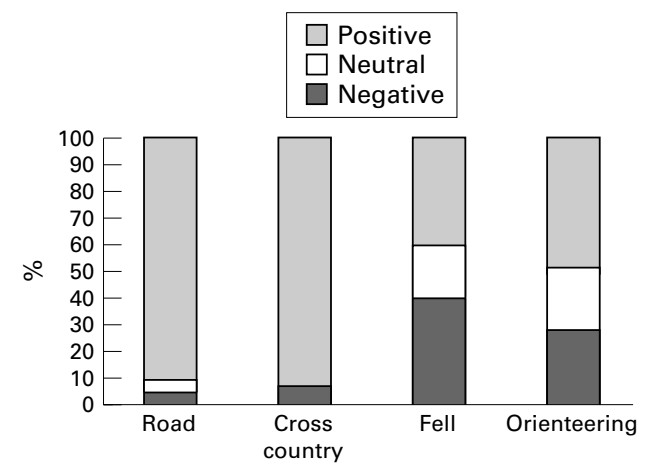

Figure 3 Differences (\%) in trends of heart rate profiles for each running event. 
Table 3 Variance of the residuals of heart rate around established regression lines

\begin{tabular}{lrrrr}
\hline & \multicolumn{3}{l}{ Variance } & \\
\cline { 2 - 5 } & Road & Cross country & Fell & Orienteering \\
\hline Mean & 3.12 & 3.35 & 14.15 & 48.51 \\
Standard & & & & \\
$\quad$ deviation & 3.13 & 3.02 & 12.44 & 30.45 \\
Minimum & 0.77 & 1.13 & 5.31 & 14.20 \\
Maximum & 15.12 & 11.60 & 61.66 & 125.23 \\
\hline
\end{tabular}

\section{Discussion}

The principal difference between the running events studied, as indicated by heart rate, was that in both orienteering and fell running there was considerable variation in heart rate during competition. The mean heart rate decreased progressively with increasingly difficult underfoot conditions; the highest mean heart rate was recorded in road running, while orienteering elicited the lowest heart rate response.

The large degree of variability observed in the heart rates during orienteering is consistent with previous findings for this sport. ${ }^{112}$ These patterns may reflect the nature of the sport and the terrain encountered. As such, the stimulus for heart rate response resembles that in fell running; energy expenditure changes continuously in these events with differing degrees of difficulty of terrain and gradient. In orienteering the challenge of navigation under physiological stress is also an important factor. Intersubject variation complicates the interpretation of the terrain effect. Rough underfoot conditions could elevate the heart rate response as the athlete overcomes the added obstruction of branches, rocks, and natural growth underfoot. Alternatively, athletes who can maintain a consistent level of effort - that is, equivalent to some physiological threshold-may reduce their pace in order to compensate for the difficult terrain. A less fit competitor may be unable to maintain a running pace in such conditions and thus the heart rate will decrease. Similarly, in terrain that is easy to run through at a fast pace, with little underfoot roughness, the heart rate may decrease as the conditions become less demanding. In experienced competitors this is less likely to occur as their pace will change in response to the change in conditions. If the subject has a high level of fitness, his/her pace will increase with a corresponding rise (or maintenance of previously elevated level) in heart rate.

The effect of gradient is well established in terms of the increased demands of uphill walking and running. Berry et $a l^{13}$ found that well trained subjects $\left(\dot{\mathrm{V}}_{2} \mathrm{MAX}>60 \mathrm{ml} / \mathrm{kg} / \mathrm{min}\right.$ ) had a higher heart rate when walking uphill than when running on the level. Downhill running $(-10 \%)$ results in a decreased heart rate for a set speed, ${ }^{14}$ and thus could account for the relatively high proportion of time spent with heart rates below 160 beats/minute on orienteering courses. Since the proportion of time spent at heart rates below this level was very low in fell runners $(3.7 \%)$, the effect of downhill running does not adequately account for the low heart rates in orienteering. The lack of any marked gradient variations within either the road or cross country races meant that no conlcusions could be drawn from those events regarding the gradient effect. Davies and Barnes, ${ }^{7}$ while claiming that the physiological cost of negative work is one fifth that of positive work, provided evidence that heart rate rises more sharply with increasing negative work than during positive work. This was later verified by Hesser et al, ${ }^{15}$ who also found that heart rate rose more steeply in response to negative than to positive work.

The technical aspect of orienteering has a unique influence on the heart rate response, not seen in the other races. The level of experience of an orienteering competitor is likely to affect his/her heart rate during competition. This is largely in terms of ability to navigate accurately, with experienced elite competitors making fewer and smaller mistakes. Mistakes generally lead to a reduction in heart rate as the competitor must stop or reduce pace to relocate. A greater percentage of low heart rates would thus be expected in inexperienced competitors. Bird et $a l^{12}$ found that, in a group of non-elite mixed age subjects (age range 15-62), heart rate was below 160 beats/minute for $54 \%$ of their course. This was in contrast with the elite group in this study who spent only $15.9 \%$ of their courses with heart rates below 160 beats/minute.

The mean heart rates measured in the orienteering competitors and fell runners in the current study were similar to those found in high level soccer players, ${ }^{16}$ reflecting the similarities in terms of the varying energy demands. A top level Swedish team member was reported to have had a mean heart rate of 175 beats/minute during a major match. This is higher than the levels recorded for less important matches, during which mean heart rates of between 157 and 165 beats/minute were recorded. ${ }^{17-19}$ Indoor exercise routines designed to stress the body aerobically have been successful in simulating the heart rates obtained in running. High impact aerobic dance incorporating arm exercise resulted in similarly high heart rates (mean 174 beats/minute).$^{20}$

Road running, on the other hand, involves a very consistent pace with little variation normally seen during a race. Garbutt et $a l^{1}$ observed male marathon runners during a 30 minute treadmill run. Their marathon race pace elicited a mean heart rate of 171 beats/minute. This is close to the levels measured in the orienteering competitors, which suggests that the overall physiological stress of orienteering resembles that of marathon running but with a greater range of heart rates displayed. The duration of the races used in the road running group studied in the current research was shorter than the orienteering events. This may be the reason for the higher mean heart rates of this group and would explain the difference in relation to the mean heart rate of the marathon runners during racing, previously discussed. The cross country races used were also relatively short, averaging about $7 \mathrm{~km}$. The fell running group had heart rates close to those of the 
orienteering competitors, and the duration of the two events was similar.

The trend of the heart rate regression lines provided information about the pacing of participants during each of the events. During straightforward races such as road and cross country races, subjects pace themselves in such a way as to be able to increase their velocity in the closing stages of the event. An awareness of and familiarity with the demands of such races allows initial sparing of energy in the early stages, followed by a gradual increase in pace in the later stages of the race. Intermediate times were recorded by some subjects during races in which distance was indicated (mainly road races), and in many cases the final mile/ $\mathrm{km}$ was the fastest one recorded (excluding the first). Departures from this trend (neutral) tended to occur in less experienced athletes who failed to pace themselves successfully and subsequently reduced their pace as they became disheartened. In both fell racing and orienteering the impending conditions and therefore demands are generally not known. This means that competitors in these events must pace themselves equally throughout the race. In fell running, however, a downhill finish is also common and may influence the profile of the heart rates. In many of the races recorded, a downhill finish did not result in a decreased heart rate since the increased velocity associated with the finishing spurt superceded the gradient effect. The necessity to maintain an exercise intensity whereby mental function is optimal ${ }^{22}$ is also an important consideration in orienteering since the competitor must continue to navigate to the finish.

The findings of this study have implications for the training of elite orienteers and fell runners. It is evident that the training regimen of these athletes should closely resemble that of an endurance road runner given the similarities shown in mean heart rates. Both disciplines, however, demand training at a high level of intensity, similar to the training required in preparation for intermittent exercise such as soccer. The fact that they experience phases of reduced heart rate shows that there is a potential in competition to recover from heavy bursts of exercise. Since there is a possibility that heart rates during running off-road may not be a direct reflection of the oxygen cost, it is important that they refer to competition heart rates in the context of the specific terrain encountered, when establishing training targets.

In conclusion, athletes taking part in offroad running events are subjected to a greater variability in exercise intensity than those participating in road races. Cross country rac- ing closely resembles road running in terms of mean exercise intensity, although there is a greater distribution of heart rates for the cross country running. Participation in orienteering results in a significantly more variable response than all other events investigated. The trend analysis, however, showed that orienteers and fell runners pace themselves successfully throughout competitions despite inconsistent conditions, while both road runners and cross country runners build up to a peak intensity towards the end of their courses.

1 Soule RG, Goldman RF. Terrain coefficients for energy cost prediction. F Appl Physiol 1972;32:706-8.

2 Zamparo P, Perini R, Orizio C, et al. The energy cost of walking or running on sand. Eur f Appl Physiol 1992;65: $183-7$

3 Rodahl K, Vokac Z, Fugelli P, et al. Circulatory strain, estimated energy output and catecholamines excretion in Norwegian coastal fishermen. Ergonomics 1974;17:585-602.

4 Clifford PS, Coast JR, Swain DP, et al. Heart rate/oxygen consumption relationship during cycling. Med Sci Sports Exerc 1986;18:S36.

5 Yamaji K, Greenley M, Northey DR, Houghson RL. Oxygen uptake and heart rate responses to treadmill and water running. Can $\mathcal{F}$ Sports Sci 1990;15:96-8.

6 Creagh U, Reilly T. Investigation of the $\mathrm{HR}-\mathrm{VO}_{2}$ relationship during uphill running. [Abstract] $\mathcal{F}$ Sports Sci 1996;14: $72-3$.

7 Davies CTM, Barnes C. Negative (eccentric) work. II. Physiological responses to walking uphill and downhill on a Physiological responses to walking uphill and downhill

8 Pimental NA, Shapiro Y, Pandolf KB. Comparison of uphill and downhill walking and concentric and eccentric cycling. and downhill walking and conce

Ergonomics 1982;25:373-80.
9 Winer BJ. Statistical principles in experimental design. New York: McGraw-Hill, 1971.

0 Ryan TA, Joiner BL. Normal probability plots and tests for normality. Technical Report, Statistics Department, The Pennsylvania State University, 1976.

11 Peck G. Measuring heart rate as an indicator of physiological stress in relation to orienteering performance. Scientific fournal of Orienteering 1990;6:26-42.

12 Bird SR, Bailey R, Lewis J. Heart rates during competitive orienteering. Br f Sports Med 1993;27:53-7.

13 Berry MJ, Weyrich AS, Robergs RA, et al. Ratings of perceived exertion in individuals with varying fitness levels perceived exertion in individuals with varying fitness levels during

14 Schwane JA, Johnson SR, Vandenakker CB, Armstrong RB. Delayed-onset muscular soreness and plasma CPK and LDH activities after downhill running. Med Sci Sports Exerc 1983;15:51-6.

15 Hesser CM, Linnarson D. Bjurstedt H. Cardiorespiratory and metabolic responses to positive, negative and minimum-load dynamic leg exercise. Respir Physiol 1977; 30:51-67.

16 Reilly T. Energetics of high-intensity exercise (soccer) with particular reference to fatigue. I Sports Sci 1997;15:25763.

17 Seliger V. Heart rate as an index of physical load in exercise. Scripta Medica 1968;41:231-40.

18 Seliger V. Energy metabolism in selected physical exercise. Internationale Zeitchrift fur Angewandte Physiologie 1968;25: 104-20.

19 Reilly T. Fundamental studies in soccer. In Andresen R, ed. Sportswissenschraft und Sportpraxis: Beitrage Zur Sportspiel Forschung. Hamburg: Verlag Ingrid Czwalina, 1986:11421

20 Bell JM, Bassey EJ. A comparison of the relation between oxygen uptake and heart rate during different styles of aerobic dance and a traditional step test in women. Eur $\mathcal{F}$ Appl Physiol 1994;68:20-4.

21 Garbutt G, Boocock MG, Reilly T, Troup JDG. Running speed and spinal shrinkage in runners with and without low back pain. Med Sci Sports Exerc 1990;22:769-72.

$22 \mathrm{Fach} \mathrm{HH}$. Visual attention and concentration during stepwise increased treadmill velocity in orienteers and long-distance runners. Scientific fournal of Orienteering $1985 ; 1: 14-23$ 